\title{
Metodología de rediseño de un robot paralelo tipo Delta de 3-GDL en función de un espacio de trabajo prescrito
}

\author{
A. Balmaceda ${ }^{{ }^{*}}$ y E. Castillo ${ }^{2}$ \\ Centro de Investigación en Ciencia Aplicada y Tecnología Avanzada \\ Instituto Politécnico Nacional \\ Querétaro, Querétaro, México \\ 1abalmacedas0800@ipn.mx 2 ecastilloca@ipn.mx
}

(Recibido/received: 09-Enero-2013; aceptado/accepted: 24-Julio-2013)

\begin{abstract}
RESUMEN
En este artículo se desarrolla y aplica una metodología de rediseño de un mecanismo paralelo tipo Delta en función de un espacio de trabajo prescrito, para cualquiera que sea su forma geométrica. La metodología se implementó en el programa computacional Matlab ${ }^{\circledR}$ sin utilizar una función objetivo, seleccionando los valores mínimos factibles de las variables de diseño, estas son las longitudes de: la plataforma fija y móvil, los eslabones o brazos que conforman la cadena cinemática y una distancia entre la base fija y el espacio prescrito. La forma geométrica del volumen prescrito fue definida en comparación con la forma del espacio de trabajo de dos robots tipo Delta disponibles en el mercado internacional. La metodología fue aplicada al robot paralelo tipo Delta Parallix LKF2040, creado en el CICATA-IPN, Querétaro, México, con el fin de diseñar su versión industrial.
\end{abstract}

Palabras claves: robot paralelo tipo Delta; espacio de trabajo; optimización.

\begin{abstract}
This paper develops and applies a methodology for re-designing a Delta-type parallel mechanism based on a prescribed workspace, for whatever geometric shape. The methodology was implemented in Matlab ${ }^{\circledR}$ software without using an objective function, selecting feasible minimum values of the design variables, these lengths are: fixed and mobile platform, links or arms that comprising the kinematic chain and a distance between the base fixed and space requirement. The geometric shape defined prescribed volume was compared with the shape of two workspace Delta-type robots available in the international market. The methodology was applied to parallel robot Delta-type Parallix LKF-2040, created in CICATA - IPN, Queretaro, Mexico, in order to design their industrial version.
\end{abstract}

Keywords: delta-type parallel robot; workspace; optimization.

\footnotetext{
* Autor para la correspondencia
} 


\section{INTRODUCCIÓN}

EL robot Parallix LKF-2040 con estructura de mecanismo paralelo tipo Delta, fue creado con un enfoque didáctico, para satisfacer las necesidades de competencia educativa y mitigar la dependencia tecnológica de las instituciones de educación superior que ofertan las carreras de Mecatrónica y sus afines.

El objetivo con el manipulador Parallix LKF-2040 es ofertarlo en su versión industrial como una mejor alternativa versus los altos costos de adquisición de unidades robóticas que deben importarse de países más industrializados, los cuales difícilmente pueden ser estudiados interiormente y conocer su diseño electromecánico.

Considerando el éxito en su versión didáctica, actualmente el robot Parallix LKF-2040 no puede ser ofertado en la industria manufacturera por su espacio de trabajo relativamente pequeño, siendo este el índice de desempeño más importante en contra parte con los manipuladores seriales (Y. Jin, et al., 2011). Por tanto, fue necesario redimensionar las variables de diseño del manipulador, a fin de obtener un espacio de trabajo resultante competitivo en tareas de manufacturación (Y. Jin, et al., 2011).

Un manipulador paralelo generalizado es un mecanismo de cadenas cinemáticas en lazo cerrado, cuyo efector final es unido a la base por varias cadenas cinemáticas independientes (J.P. Merlet, 2006). Estos han sido utilizados en la industria desde el siglo pasado. Entre los utilizados están la plataforma de Gough octaedro hexápodo (1954), la plataforma de Stewart de 6-GDL y el robot paralelo tipo Delta creado por Clavel en 1991 (J.P. Merlet, 2006).

En lo que respecta a los requerimientos de necesidad, hay dos tipos de formulación del problema de diseño. Uno es encontrar la geometría de un manipulador paralelo que maximice el espacio de trabajo, sin embargo esto puede ser no tan bueno para un diseño práctico por sus características cinemáticas complejas, como se concluye en (R. Stamper, 1997) y (Y. Lou, et. al, 2007). La otra formulación está en función de un volumen deseado. Por tanto, en este trabajo la síntesis dimensional se formuló generando un manipulador con un espacio de trabajo resultante en función de un espacio de trabajo prescrito.

Una síntesis para la optimización del espacio de trabajo utilizando un algoritmo genético, fue realizado por $\mathrm{M}$.
Arsenault, y R. Boudreau (2004), considerando 3 criterios de diseño del robot paralelo: la optimización del espacio de trabajo aproximado a uno prescrito, la maximización de la destreza y la prevención de singularidades dentro del espacio resultante (M. Arsenault, 2004). Para la optimización del espacio de trabajo del CaPaMan (E. Ottaviano, 2002), se prescribió un espacio de trabajo deseable $\mathrm{V}$ ' conteniendo un paralelepípedo $\mathrm{V}^{*}$, a fin de simplificar la evaluación del volumen de trabajo y encontrar las medidas de los parámetros de diseño óptimo usando el Toolbox de Optimización de Matlab®.

Otra síntesis dimensional en base a un espacio de trabajo prescrito en forma de cubo, se desarrolló con el análisis geométrico directo e indirecto de un robot paralelo tipo Delta. Aquí se obtuvo una función objetivo basada en el concepto matemático de potencia de un punto, la cual se optimizó mediante un algoritmo genético ejecutado en Matlab® (M. A. Laribi, 2006).

Y. Jin en 2011 propuso un enfoque de partición finita para partición paramétrica y proporcional basado en propiedades de topología de $\mathrm{SE}(3)$, a fin de integrar el espacio de trabajo completo de manipuladores paralelos por medio de simples sumatorias de los elementos de volumen base de $\mathrm{SE}(3)$. Se utilizaron la razón de volumen de espacio de trabajo y el índice de condicionamiento global como índices de desempeño para la optimización de un manipulador 3RP PS usado como ejemplo.

En (Y.J. Lou, 2005) se propuso una nueva optimización para el diseño de manipuladores paralelos, maximizando la forma regular del espacio de trabajo prescrito sujeta a restricciones de destreza. Se utilizó la técnica llamada búsqueda aleatoria controlada, que consiste en seleccionar un punto de solución por selección aleatoria de una distribución de probabilidad normal centrada en el mejor valor previo.

En este artículo se realiza una síntesis dimensional bajo restricciones sin utilizar una función objetivo para simplificar el proceso de rediseño. De un universo de soluciones óptimas calculado, se seleccionan los valores mínimos factibles de las variables de diseño. La geometría del espacio de trabajo prescrito es comparable al de dos robots tipo Delta del mercado internacional. 


\section{CINEMÁTICA DEL ROBOT TIPO DELTA}

\section{Modelo geométrico directo}

Llámese a un robot paralelo, un robot de al menos 2 cuerpos conectados por más de una cadena cinemática. Una cadena cinemática es un ensamble de segmentos unidos con articulaciones (Clavel, 1991).

El robot paralelo de configuración tipo Delta estudiado tiene 3 GDL y consiste de una plataforma fija con forma de triángulo equilátero, cuyo apotema tiene una longitud $r$, unida a una plataforma móvil a través de 3 cadenas cinemáticas movidas por motores instalados en la base fija que transmiten el movimiento a la base móvil de longitud rpm. Véase Figura 1.

Cada brazo está constituido por 2 eslabones, 1 articulación rotacional y 2 universales (manipulador paralelo 3RUU). El primer eslabón de longitud La es una sola pieza y el segundo de longitud Lb está formado por barras paralelas (Clavel, 1991). Los ángulos de giro de los brazos se definen como: $\alpha 1 \mathrm{i}, \alpha 2 \mathrm{i}, \alpha 3 \mathrm{i}$, así como el ángulo entre motores en la base fija es $\theta_{\mathrm{i}}(\mathrm{i}=1,2,3)$ y sus valores son: $\theta_{1}=30^{\circ}, \theta_{2}=150^{\circ}, \theta_{3}=270^{\circ}$. (Véase Fig. 1).

Sea $\mathrm{P}$ el punto de coordenadas $\left[\mathrm{x}_{\mathrm{p}}, \mathrm{y}_{\mathrm{p}}, \mathrm{z}_{\mathrm{p}}\right]$ el centro de la base móvil del manipulador, entonces el modelo geométrico puede escribirse como:

$\left[\left(R+L a \cos \alpha_{1 i}\right) \cos \theta_{i}-x_{p}\right]^{2}+\left[\left(R+L a \cos \alpha_{1 i}\right) \operatorname{sen} \theta_{i}-\right.$ $\left.y_{p}\right]^{2}+\left[- \text { Lasen }_{1 i}-z_{p}\right]^{2}-L b^{2}=0$

La ecuación (1) se elevó al cuadrado para eliminar las variables de las articulaciones pasivas, donde: $\mathrm{R}=\mathrm{r}-$ rpm y de aquí en adelante $\alpha_{1 \mathrm{i}}$ llámese $\alpha_{\mathrm{i}}$ para $\mathrm{i}=1,2,3$, para simplificar la nomenclatura.

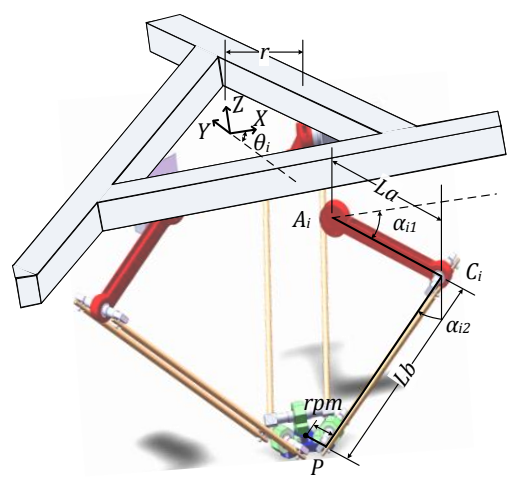

a)

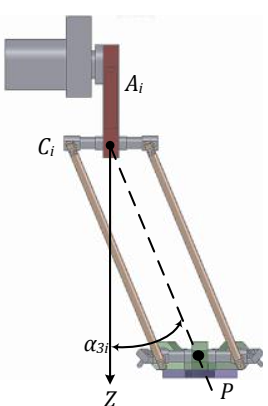

b)
Figura. 1. Parámetros del robot Delta Parallix LKF2040: a) Parámetros cinemáticos del mecanismo. b) Formación del ángulo $\alpha_{3 i}$ en la cadena cinemática.

\section{Espacio de trabajo por modelo geométrico inverso.}

Desarrollando el modelo geométrico inverso se tiene que las coordenadas del punto $C_{i}$ (ver Fig. 1) son (M. Velázquez, 2003):

$$
C_{i}=\left[\begin{array}{c}
R+\operatorname{Lacos}_{i} \\
0 \\
-\operatorname{Lasen}_{i}
\end{array}\right]
$$

Considerando la rotación $\theta_{\mathrm{i}}$ alrededor del eje $\mathrm{Z}$ y ya que $C_{i}$ pertenece a una esfera:

$$
\left(X \cos \theta_{i}-x_{p}\right)^{2}+\left(X \operatorname{sen} \theta_{i}-y_{p}\right)^{2}+\left(Z-z_{p}\right)^{2}=L b^{2}
$$

De esta manera, las incógnitas de la ecuación (3) son los ángulos $\alpha_{\mathrm{i}}$. Entonces desarrollando los binomios factorizando y sustituyendo $\mathrm{X}=\mathrm{R}+\mathrm{Lacos} \alpha_{\mathrm{i}}, \quad \mathrm{Z}=$ -Lasen $\alpha_{\mathrm{i}}$ y simplificando se obtiene:

$2 R L \operatorname{Lacos} \alpha_{i}-L a \cos \alpha_{i}\left(2 x_{p} \cos \theta_{i}+2 y_{p} \cos \theta_{i}\right)+2 z_{p}$ Lasen $_{i}=$ $L b^{2}-L a^{2}-R^{2}-x_{p}^{2}-y_{p}^{2}-z_{p}^{2}+R\left(2 x_{p} \cos \theta_{i}+2 y_{p} \cos \theta_{i}\right)$

Resolviendo algebraicamente para $\alpha_{\mathrm{i}} \mathrm{y}$ sustituyendo por $\mathrm{q}_{\mathrm{i}}=2 \mathrm{x}_{\mathrm{p}} \cos \theta_{\mathrm{i}}+2 \mathrm{y}_{\mathrm{p}} \cos \theta_{\mathrm{i}}$ y $\mathrm{s}=\frac{1}{\mathrm{La}}\left(\mathrm{Lb}^{2}-\mathrm{La}^{2}-\mathrm{R}^{2}-\right.$ $\mathrm{x}_{\mathrm{p}}^{2}-\mathrm{y}_{\mathrm{p}}^{2}-\mathrm{z}_{\mathrm{p}}^{2}$ ) se tiene finalmente:

$\tan \frac{\alpha_{i}}{2}=\frac{-2 z_{p} \pm \sqrt{4 z_{p}^{2}+4 R^{2}-s^{2}-q_{i}\left(4 R+\frac{2 s R}{L a}\right)+q_{i}{ }^{2}\left(1-\frac{R^{2}}{L a^{2}}\right)}}{-2 R-s-q_{i}\left(\frac{R}{L a}-1\right)}$

Por tanto, la ecuación (5) resuelve la cinemática inversa del mecanismo encontrando los ángulos $\alpha_{\mathrm{i}}$ de los puntos 
alcanzables en su espacio de trabajo, a través de un algoritmo en Matlab ${ }^{\circledR}$.

\section{Límites geométricos del espacio de trabajo}

El espacio de trabajo del robot Delta se define como la región en el plano cartesiano de tres dimensiones, que puede ser alcanzada por un punto de la plataforma móvil (M. A. Laribi, 2006). En el cálculo se ha considerado la longitud de las cadenas cinemáticas, el rango de giro máximo y mínimo de los $\alpha_{\mathrm{i}}$ y el alcance máximo de la base móvil en el eje $\mathrm{z}$.

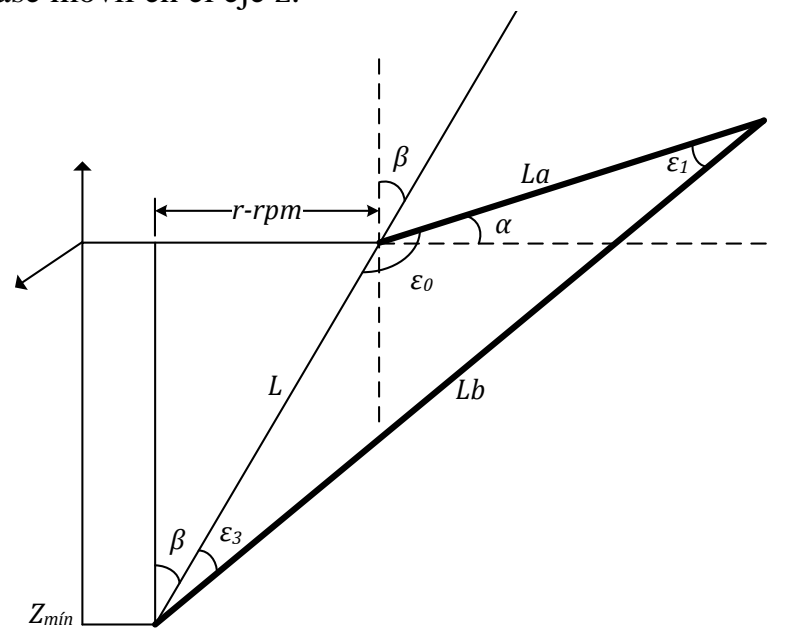

Figura. 2. Diagrama geométrico de cálculo z máxima y mínima (M. Velázquez, 2003).

En el mecanismo hay posiciones de la base móvil que no deben ocurrir para evitar colisiones y bloqueos mecánicos entre los brazos, estas condiciones están sujetas al valor del ángulo $\varepsilon_{1} \approx 90^{\circ}$ (ver Fig. 2).

Si se tiene que $\lim _{\varepsilon_{1} \rightarrow 0} \mathrm{~L}=\sqrt{\mathrm{Lb}^{2}+\mathrm{La}^{2}-2 \mathrm{LaLb}}$ y el $\lim _{\varepsilon_{1} \rightarrow 0} \varepsilon_{0}=180^{\circ}$, entonces $\alpha+\beta=90^{\circ}$. Y además, $\beta=\operatorname{arcsen}\left(\frac{\mathrm{R}}{\mathrm{L}}\right)$, por lo tanto: $\alpha_{\operatorname{mín}}=\frac{\pi}{2}-\beta$. De manera que si $\alpha_{\mathrm{i}}$ es inferior a $\alpha_{\text {mín }}$, entonces ocurrirá una colisión o bloqueo mecánico en la estructura paralela.

De la Figura 2, si se despeja para $\mathrm{Z}_{\text {mín }}$ se tiene que,

$Z_{\text {min }}=L \cos \beta$

Ahora, para cuando $\alpha_{\mathrm{i}}$ toma el valor de $\alpha_{\mathrm{i}}=90^{\circ} \mathrm{y}$ $\mathrm{Z}_{0}=\sqrt{\mathrm{Lb}^{2}-(\mathrm{r}-\mathrm{rpm})^{2}}$ entonces (ver Figura 3).

$Z_{\text {máx }}=L a+Z_{0}$

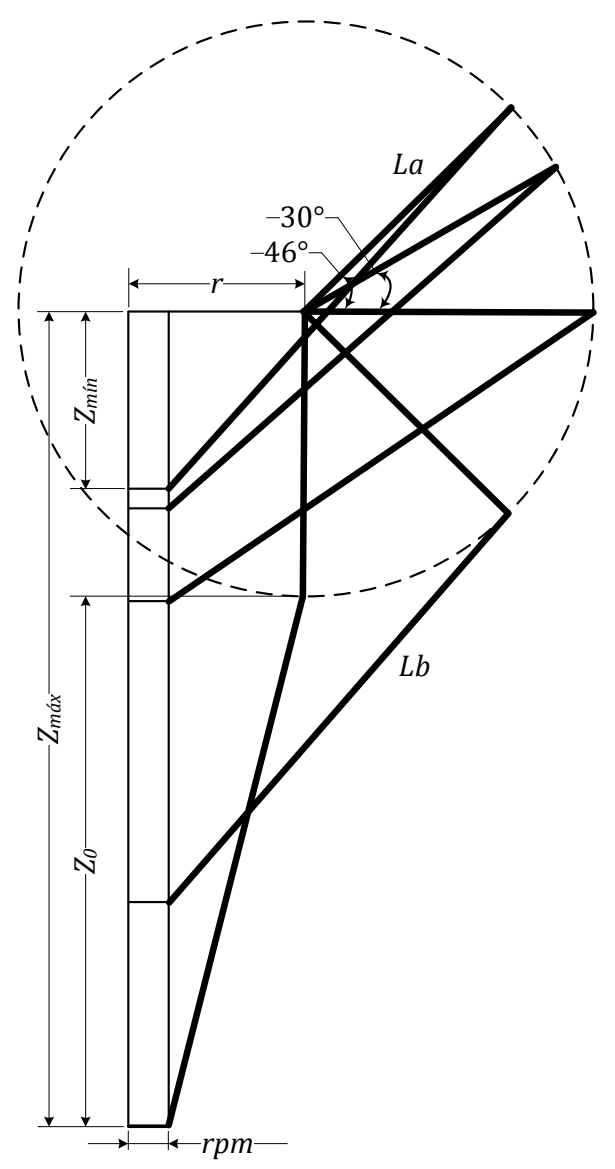

Figura. 3. Desplazamiento máximo del mecanismo en el eje Z (M. Velázquez, 2003).

\section{SÍNTESIS DIMENSIONAL DEL MANIPULADOR.}

El objetivo aquí es generar un manipulador con un espacio de trabajo resultante $\left(\mathrm{W}_{\mathrm{r}}\right)$, que contenga un espacio de trabajo prescrito $\left(\mathrm{W}_{\mathrm{p}}\right)$ representado por un poliedro.

En el mercado internacional se ofertan robots paralelos tipo Delta para la industria. El robot Parallix LKF-2040 debe obtener nuevas dimensiones para alcanzar un espacio de trabajo, el cual se prescribe mediante la semejanza con los espacios de trabajo de dos robots tipo Delta industriales ofertados en el mercado internacional.

Uno de los robots, que se utilizó como proyección de dimensiones del espacio de trabajo prescrito para el nuevo Parallix, es el robot Delta Adept Quatttro s650H de la compañía Adept Technology, Inc. de los Estados Unidos de América. 
Tabla 1

Comparación del Parallix lkf-2040 con robots del mercado y su nueva versión

\begin{tabular}{lcccc}
\hline \hline Item & $\begin{array}{c}\text { Adept } \\
\text { Quatro } \\
\text { s650H }\end{array}$ & $\begin{array}{c}\text { IRB 360- } \\
3 / 1130 \\
\text { FlexPicker }\end{array}$ & $\begin{array}{c}\text { Parallix } \\
\text { LKF-2040 }\end{array}$ & $\begin{array}{c}\text { Parallix } \\
\text { nueva } \\
\text { versión } \\
\left(W_{p}\right)\end{array}$ \\
\hline $\begin{array}{l}\text { Alcance } \\
\text { en eje z }\end{array}$ & $500 \mathrm{~mm}$ & $300 \mathrm{~mm}$ & $250 \mathrm{~mm}$ & $350 \mathrm{~mm}$ \\
$\begin{array}{l}\text { Alcance } \\
\text { en eje } x \text { e }\end{array}$ & $1,300 \mathrm{~mm}$ & $1,130 \mathrm{~mm}$ & $600 \mathrm{~mm}$ & $1,040 \mathrm{~mm}$ \\
$\begin{array}{l}\text { Número } \\
\text { de brazos }\end{array}$ & 4 & 4 & 3 & 3 \\
$\begin{array}{l}\text { Compañía } \\
\text { Institución }\end{array}$ & $\begin{array}{c}\text { Adept } \\
\text { Technology } \\
\text { Inc. }\end{array}$ & $\begin{array}{c}\text { ABsea } \\
\text { Brown } \\
\text { Boveri) } \\
\text { Robotics }\end{array}$ & $\begin{array}{c}\text { Politécnico } \\
\text { Nacional }\end{array}$ & $\begin{array}{c}\text { Politécnico } \\
\text { Nacional }\end{array}$ \\
País & $\begin{array}{c}\text { Estados } \\
\text { Unidos }\end{array}$ & $\begin{array}{c}\text { Suecia } \\
\text { México }\end{array}$ & México
\end{tabular}

Otro robot paralelo Delta con el que se podrá asemejar el volumen prescrito del Parallix redimensionado, es el IRB 360-3/1130 de FlexPickerTM de Suecia, el cual tiene un espacio de trabajo de $1,130 \mathrm{~mm}$ de diámetro para $\mathrm{x}$ e $\mathrm{y}$, en una altura de $300 \mathrm{~mm}$ sobre el eje $\mathrm{z}$.

En la Tabla 1 el robot Parallix LKF-2040 es comparado, y puede verse a dónde está y a dónde se quiere ir, con respecto a los dos robots Delta ofertados en el mercado internacional.

Con los datos de la Tabla 1, es posible representar geométricamente el espacio de trabajo prescrito (ver Fig. 4) y compararse con el espacio de trabajo actual del manipulador Parallix LKF-2040.

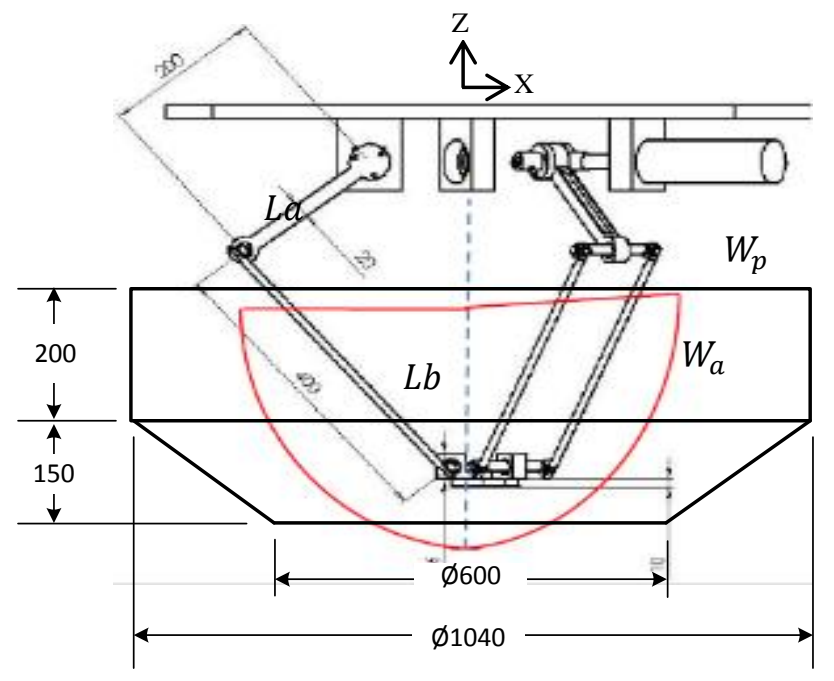

Figura. 4. Espacio de trabajo actual $\left(W_{a}\right)$ y prescrito del robot Parallix.
Análisis de sensibilidad dimensional del robot Parallix LKF-2040.

Un análisis de sensibilidad tiene que ver con la obtención de información sobre el comportamiento de la posible solución, cuando el modelo sufre ciertos cambios de parámetros. Se necesita en especial dicho análisis para establecer las variables críticas de diseño cuando no se pueden estimar con exactitud (H. Taha, 2004). Por tal razón, se hicieron simulaciones del $W_{r}$ variando los valores de $\mathrm{r}$, La y Lb del robot Parallix LKF-2040 (ver Fig. 1) resolviendo su cinemática inversa en Matlab ${ }^{\circledR}$.

La primera simulación se hizo variando las dimensiones de la plataforma fija del Parallix LKF-2040, que separa a cada motor por su apotema de longitud $r$ (ver Fig. 3). A continuación se presenta la Figura 5 con los volúmenes de los espacios de trabajo resultantes de las variaciones dimensionales de la variable de diseño $r$.

Los volúmenes de los espacios de trabajo alcanzables por la plataforma móvil del robot representados con cruces (+), se reducen considerablemente mientras se incrementa la longitud $r$ (ver Fig. 5) y esto no es conveniente para el robot de versión industrial. Por lo tanto la variación de dimensiones solamente de la plataforma fija del Parallix, no es crítica para alcanzar el espacio de trabajo prescrito.

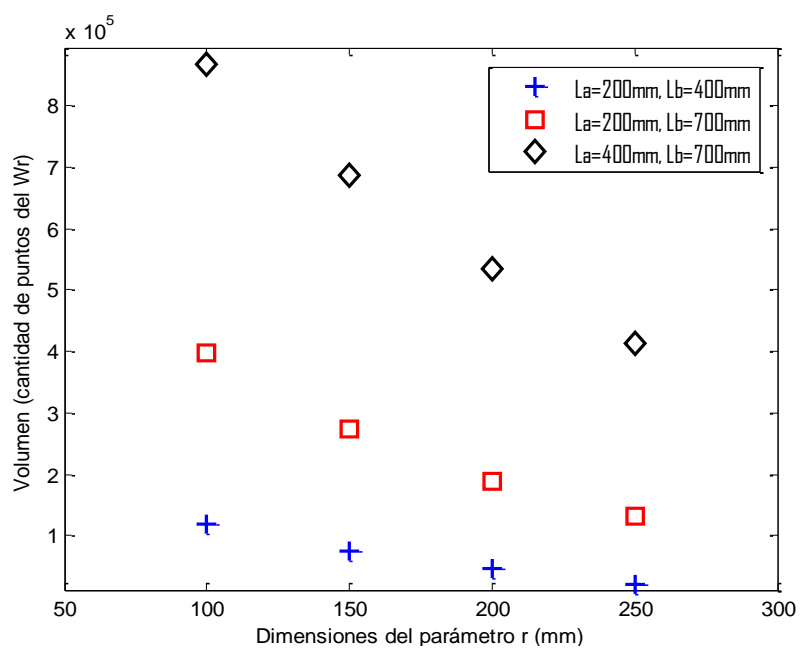

Figura. 5. Volúmenes de espacios de trabajo para $\mathrm{r}=$ $100 \mathrm{~mm}, 150 \mathrm{~mm}, 200 \mathrm{~mm}$ y $250 \mathrm{~mm}$.

Se hicieron más simulaciones pero ahora variando dimensiones de los eslabones del robot, además de las mismas variaciones de $r$, estos son los volúmenes marcados por cuadros rojos $(\square)$ con $\mathrm{La}=200 \mathrm{~mm}, \mathrm{Lb}=$ $700 \mathrm{~mm}$ y los señalados con rombos negros $(\diamond)$ para $\mathrm{La}=$ 
400mm, Lb= 700mm. En estos casos (ver Fig. 5), el espacio de trabajo también tiende reducirse en lo ancho $\mathrm{y}$ alto del alcance del manipulador a pesar de los incrementos de la longitud de los eslabones.

Una simulación más fue hecha variando sólo la longitud $\mathrm{La}$, con $\mathrm{Lb}=700 \mathrm{~mm}, \mathrm{r}$ y rpm con $150 \mathrm{~mm}, 50 \mathrm{~mm}$ respectivamente. Nótese que (ver Fig. 6) cuando $\mathrm{La}=$ $600 \mathrm{~mm}$ el volumen de trabajo es menor al de $\mathrm{La}=$ $400 \mathrm{~mm}$, esto significa que si el primer eslabón es relativamente tan grande como el segundo, entonces existirán más colisiones entre las cadenas cinemáticas limitando el alcance del robot.

Por estas razones, es difícil establecer las variables críticas de diseño debido al comportamiento no lineal del problema de redimensionamiento. De modo que es necesario considerar más parámetros de diseño y calcular sus longitudes mínimas, a fin de alcanzar el espacio de trabajo prescrito para la nueva versión industrial del Parallix.

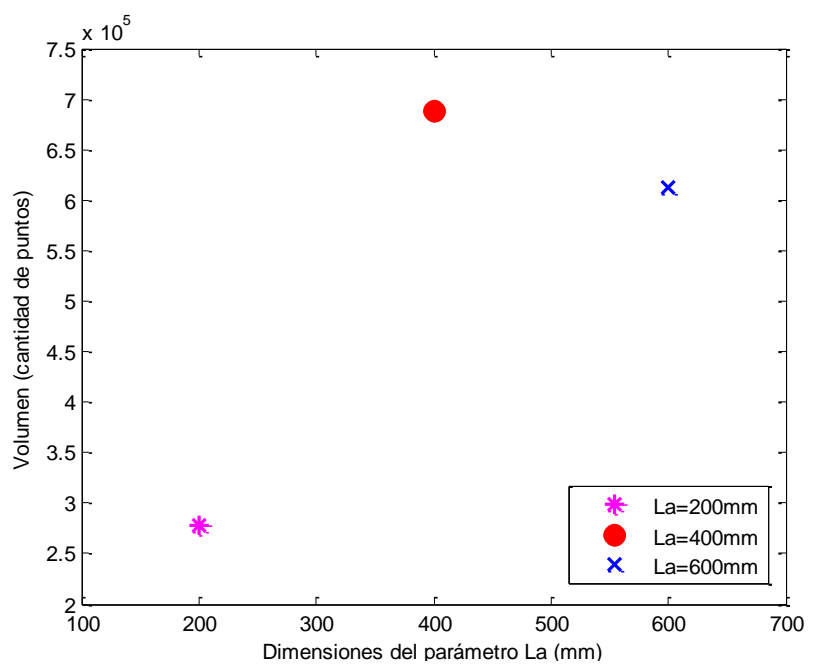

Figura 6. Volúmenes de espacios de trabajo para $\mathrm{La}=200 \mathrm{~mm}, 400 \mathrm{~mm}, 600 \mathrm{~mm}, \mathrm{r}=150 \mathrm{~mm}$ y $\mathrm{Lb}=$ $700 \mathrm{~mm}$.

\section{Formulación del problema}

Para que la geometría del $\mathrm{W}_{\mathrm{p}}$ esté contenida en el $\mathrm{W}_{\mathrm{r}}$ de la nueva versión del manipulador, es necesario redimensionar las variables de diseño del mecanismo haciendo un barrido en un rango definido, con el objetivo de obtener un universo de soluciones que cumplan con dicha condición. Si se cuenta con las dimensiones del volumen deseado en el espacio $[\mathrm{x}, \mathrm{y}, \mathrm{z}]$, entonces es posible encontrar los parámetros dimensionales mínimos del robot que lo contengan.

El problema de redimensionamiento está en función de un vector desconocido de parámetros $\mathrm{I}=\left[\mathrm{x}_{1}, \mathrm{x}_{2}, \ldots, \mathrm{x}_{\mathrm{n}}\right]$, $y x_{j} \in\left[x_{j m i n}, x_{j m a ́ x}\right], j=1,2, \ldots, n$ especifica el rango de barrido establecido para cada variable, donde $n$ indica el número de la variable de diseño n-ésima a utilizar. Aquí, la solución a este problema se denota como I'.

En este trabajo, $\mathrm{n}=5 \mathrm{y}$ el vector I está definido como:

$I=\left[\begin{array}{lllll}L a & L b & r & r p m & h\end{array}\right]$

sujeto a

$\begin{array}{ll}g_{1}: & L a<L b \\ g_{2}: & r \geq r p m \\ g_{3}: & \forall P^{k} \in W_{p}\end{array}$

donde, $\mathrm{h}$ es la distancia de separación sobre el eje $\mathrm{z}$ entre el espacio de trabajo prescrito y la plataforma fija del manipulador, y $\mathrm{P}^{\mathrm{k}}=\left[\mathrm{X}_{\mathrm{p}}^{\mathrm{k}}, \mathrm{Y}_{\mathrm{p}}^{\mathrm{k}}, \mathrm{Z}_{\mathrm{p}}^{\mathrm{k}}\right], \quad \mathrm{k}=$ $1, \ldots, N$ son los $N$ vértices coordenados de $W_{p}$.

La siguiente desigualdad plantea como restricción, que cada elemento del vector I tomará valores de un rango definido bajo criterio del usuario de esta metodología.

$g_{4}: \quad x_{j \min } \leq x_{j} \leq x_{j m a ́ x}$

El objetivo aquí, es encontrar el conjunto de parámetros factible $I^{\prime}=\left[\begin{array}{lllll}\mathrm{La} & \mathrm{Lb} & \mathrm{r} & \mathrm{rpm} & \mathrm{h}\end{array}\right]$ más pequeño.

\section{Procedimiento empleado}

Para desarrollar esta metodología deben generarse todos los posibles conjuntos de solución con los parámetros dimensionales del vector I. Esto es posible primeramente, definiendo la geometría del espacio de trabajo prescrito. Aquí fue utilizado un paralelepípedo con base trapezoidal (Fig. 7) con $\mathrm{k}=1, \ldots, 12$ puntos coordenados en el espacio $[\mathrm{x}, \mathrm{y}, \mathrm{z}]$.

Deben ser definidos los rangos e incrementos de barrido de las variables, puesto que son importantes en la generación del universo de soluciones. Estos dependen 
del criterio del usuario en función de la exactitud con que se desea alcanzar el espacio de trabajo prescrito y del tamaño de este.

Los rangos e incrementos se expresan respectivamente así:

$$
\begin{aligned}
& x_{j_{\text {mín }}} \leq x_{j}\left(\Delta_{x_{j}}\right) \leq x_{j_{\text {máx }}} \\
& \Delta_{x_{j}}=x_{j_{(i+1)}}-x_{j_{(i)}}
\end{aligned}
$$

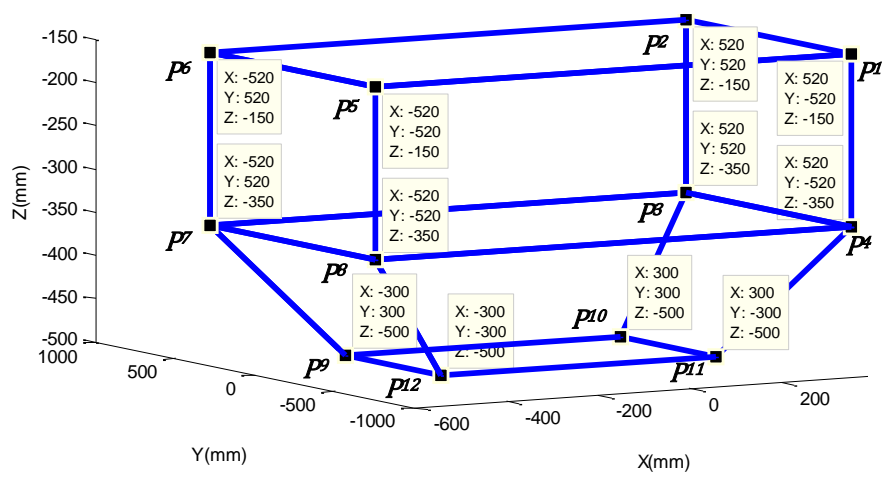

Figura 7. Geometría y coordenadas del $W_{p}$ en el espacio $[x, y, z]$.

Definidos los rangos e incrementos de cada variable del

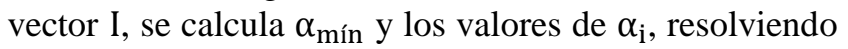
la cinemática inversa del mecanismo con la ecuación (5).

Se comprueban que todos los $\alpha_{i} \in \alpha_{\text {mín }} \leq \alpha_{i} \leq \alpha_{\text {máx }}$, $\mathrm{i}=1, \ldots, 3$ para cada $\Delta_{\mathrm{x}_{\mathrm{j}}}$. Cuando esto es verdadero, se verifica que el conjunto de combinaciones de solución obtenido satisface las restricciones (10) y (11). Si esto es así, se produce con dichos valores la matriz $\mathrm{S}_{\mathrm{mxn}}(\mathrm{m}$, indica el número del m-ésimo conjunto de solución generado).

$S=\left[\begin{array}{ccc}x_{11} & \cdots & x_{1 n} \\ \vdots & \ddots & \vdots \\ x_{m 1} & \cdots & x_{m n}\end{array}\right]$

En Matlab ${ }^{\circledR}$ fue desarrollado el código que resuelve la cinemática del mecanismo (ver Figura 8), ciclado con los rangos de las $\mathrm{x}_{\mathrm{j}}$ para encontrar todas las combinaciones de solución de las variables factibles.

El próximo paso en la metodología es el cálculo de las longitudes mínimas de las variables de diseño y el código de programación para esto, fue hecho en $\mathrm{Matlab}^{\circledR}$ (ver Fig. 9).

Del conjunto de soluciones (15) calculado inicialmente, se extraen las combinaciones donde La satisface la restricción (9), para generar la matriz $\mathrm{M} 1_{\mathrm{qxn}}$ que contiene todas las q combinaciones correspondientes al valor mínimo de La.

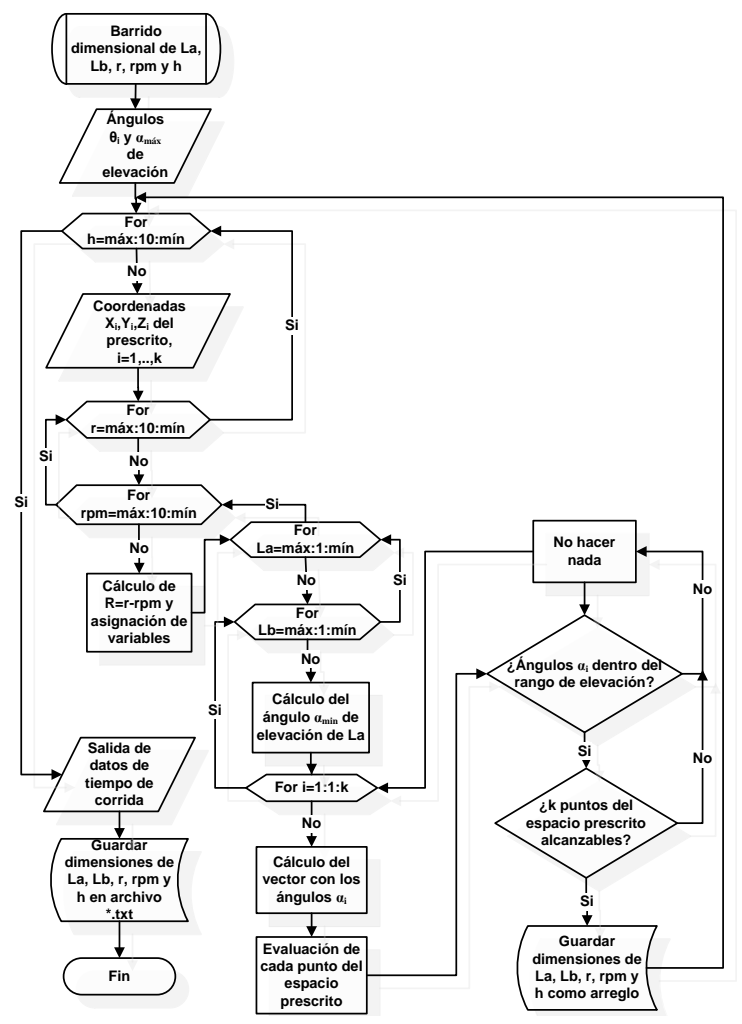

Figura 8. Diagrama de flujo del barrido de variables de diseño del robot.

Seguidamente, de la matriz $\mathrm{M} 1_{\mathrm{qxn}}$ se obtienen las $\mathrm{t}$ combinaciones pertinentes al valor mínimo de Lb para crear la matriz $\mathrm{M} 2_{\mathrm{txn}}$. Análogamente, se producen las matrices $\mathrm{M}_{\mathrm{sxn}}$ y $\mathrm{M} 4_{\mathrm{vxn}}$, que contienen los valores mínimos de $\mathrm{r}$ y rpm, respectivamente. Por lo tanto, de la última matriz se selecciona el valor mínimo de $\mathrm{h}$ y esta combinación final genera el vector de solución I'.

Para la aplicación de la metodología de rediseño en este trabajo, el $\mathrm{W}_{\mathrm{p}}$ fue definido en comparación al de dos robots tipo Delta mencionados anteriormente, a fin de que el nuevo Parallix pueda ser competitivo en base a un mayor volumen de trabajo alcanzable y ser ofertado en el mercado nacional e internacional. 
Las dimensiones establecidas del $\mathrm{W}_{\mathrm{p}} \mathrm{y}$ las $\mathrm{k}$ coordenadas del mismo pueden verse en la Fig. 4 y 7 , respectivamente. Los rangos e incrementos definidos aquí con (13) y (14) son:

$$
\begin{gathered}
200 \leq \operatorname{La}\left(\Delta_{L a}=1\right) \leq 300 \\
700 \leq L b\left(\Delta_{L b}=1\right) \leq 790 \\
100 \leq r\left(\Delta_{r}=10\right) \leq 250 \\
30 \leq r p m\left(\Delta_{r p m}=10\right) \leq 300 \\
150 \leq h\left(\Delta_{h}=10\right) \leq 200
\end{gathered}
$$

Con la ejecución de los algoritmos (ver Fig. 8 y 9) se genera todo el conjunto solución y se seleccionan los valores mínimos para cada variable. Así, la solución está dada por el vector:

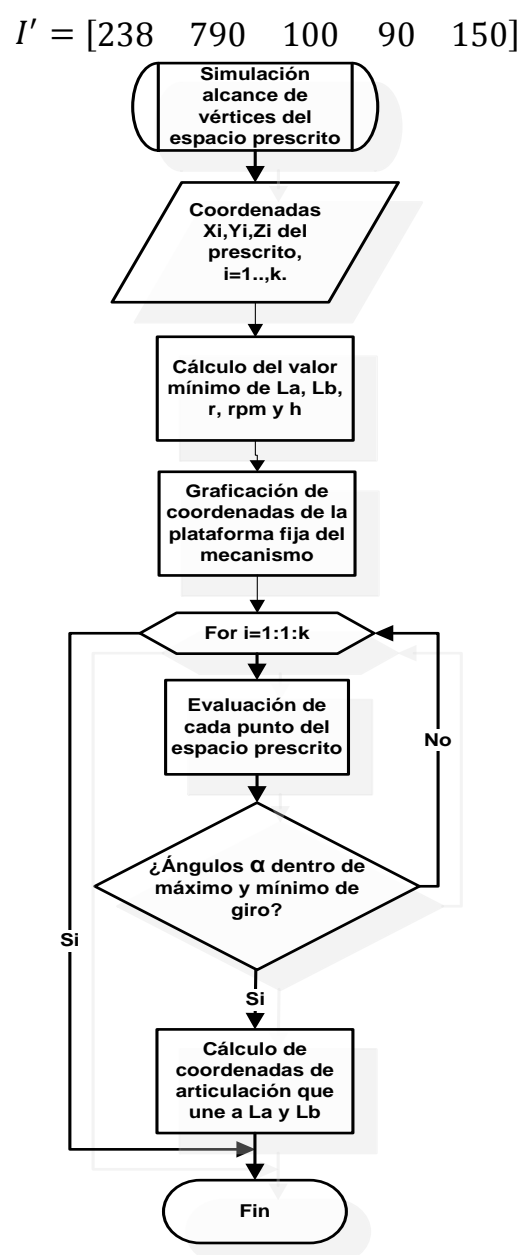

Fig. 9. Diagrama de flujo de cálculo de longitudes mínimas.
Al final el código en el programa computacional Matlab® hace una simulación del manipulador alcanzando todos los vértices $\mathrm{P}^{\mathrm{k}}$ pertenecientes al $\mathrm{W}_{\mathrm{p}}$.

\section{RESULTADOS Y DISCUSIÓN}

El paraboloide que representa el espacio de trabajo resultante del robot tipo Delta tiene dimensiones superiores en comparación con el espacio prescrito, de tal forma que la región deseada está ampliamente contenida. Entonces, ¿Esto quiere decir que la metodología no es capaz de calcular las dimensiones exactas o semejantes del manipulador para el poliedro del ejemplo de aplicación utilizado en este trabajo?

La metodología de rediseño desarrollada aquí, calcula las longitudes mínimas de las variables de diseño seleccionadas, que un mecanismo paralelo tipo Delta debe tener para contener el espacio de trabajo prescrito representado o contenido en cualquier figura geométrica.

Además, a causa de la configuración mecánica de diseño de este manipulador tipo Delta, no es conveniente que los vértices del volumen prescrito estén exactamente en los límites del espacio resultante (inclusive cuando tenga dimensiones optimizadas). Esto significaría que el robot tiene que estar en una posición extrema (sobre los límites del espacio de trabajo) para ser capaz de alcanzar esos puntos, como se concluyó en (M. A. Laribi, 2006).

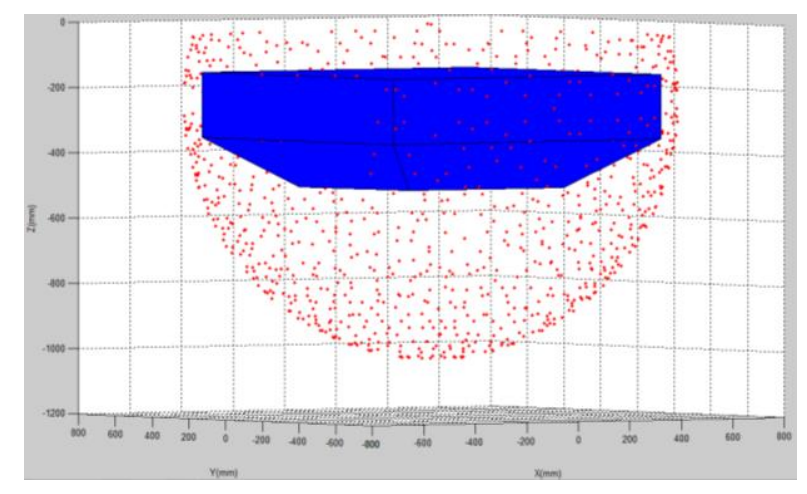

Figura 10. Espacio de trabajo prescrito inscrito en el espacio de trabajo del mecanismo redimensionado en el espacio $\mathrm{x}-\mathrm{y}-\mathrm{z}$.

Tampoco pueden dejar de existir espacios (holguras) entre los lados del prescrito y el volumen resultante (ver Fig. 10), puesto que el paraboloide es la forma geométrica que este tipo de manipulador tiene como espacio de trabajo alcanzable. 
Entonces, ¿Con la metodología no es posible redimensionar el mecanismo de tal manera que se pueda obtener un espacio de trabajo resultante que se ajuste con mayor precisión a todo el volumen prescrito?

La precisión espacial entre el volumen de trabajo resultante y el espacio de trabajo prescrito, dependerá significativamente de la figura geométrica que contenga o represente a dicho espacio deseado. Esto quiere decir, que si la geometría prescrita es semejante al paraboloide del mecanismo, entonces es posible que las holguras entre los dos volúmenes se minimicen significativamente, utilizando eficientemente la configuración mecánica de este robot tipo Delta.

Por lo tanto, la definición geométrica del espacio prescrito es una condición no restrictiva, que debe considerarse para la aplicación de esta metodología de rediseño para manipuladores tipo Delta.

Además, para que el espacio prescrito esté exactamente inscrito en el volumen resultante, basta que los incrementos de los rangos sean relativamente muy pequeños para obtener esta precisión espacial. Pero recuérdese que existirán posiciones extremas y esto no es deseable para aplicaciones industriales.

Con base a lo anterior, ¿La metodología tiene desventajas por causa de la configuración mecánica de los mecanismos tipo Delta?

No es considerable llamar desventajas por causa de las condiciones mecánicas de los robots tipo Delta. El espacio prescrito puede estar sujeto también a restricciones geométricas, de manera que estas permitan diseñar un volumen prescrito semejante al paraboloide y así utilizar con mayor eficiencia el volumen del espacio de trabajo resultante del manipulador.

Sin embargo, si el volumen prescrito se somete a restricciones geométricas, entonces limitaría la metodología de rediseño de calcular las longitudes mínimas adecuadas para el robot, en función de un espacio prescrito para cualquiera que sea su representación geométrica.

Actualmente la versión industrial está construida y ensamblada, y la programación del control de los actuadores ya fue realizada en el CICATA - IPN, Querétaro, México.

\section{CONCLUSIONES}

En este artículo se desarrolló y aplicó una metodología de rediseño para redimensionar el manipulador tipo Delta Parallix LKF-2040 en función de un espacio trabajo prescrito propuesto. El objetivo fue obtener su nueva versión industrial respecto a un volumen de trabajo competitivo alcanzable, para que pueda ser comercializado nacional e internacionalmente.

El problema de redimensionamiento fue resuelto sin utilizar una función objetivo, con el fin de simplificar el proceso de rediseño del manipulador. De un conjunto de soluciones factibles calculado bajo restricciones, se seleccionaron las longitudes mínimos de cada variable de diseño propuesta.

La discretización de la solución encontrada aquí es una característica significativa en la optimización de las variables, ya que las longitudes óptimas dependerán de los incrementos en cada rango. Así, dicha solución será entonces factible y de calidad según el tamaño de estos.

La restricción que sometía a la longitud La menor que Lb fue planteada, para que los actuadores del manipulador sean relativamente pequeños y por lo tanto de menor costo.

\section{AGRADECIMIENTOS}

Al Instituto de Ciencia y Tecnología del Distrito Federal (ICyTDF), por el apoyo económico para desarrollar este trabajo.

\section{REFERENCIAS}

Merlet, J. P., (2006). Parallel Robots, Second Edition, Springer, pp. 1-20.

Y. Jin, I.-M. Chen, G. Yang (2011). Workspace evaluation of manipulators through finite-partition of $S E(3)$. Robotics and Computer-Integrated Manufacturing, 27, pp. 850-859.

Stamper, R. E., (1997). A Three Degree of Freedom Parallel Manipulator with Only Translational Degrees of Freedom (Ph.D. Thesis). Universidad de Maryland, pp. $1-5$ y $51-64$.

Y. Lou, G. Liu, Z. Li, (2007). Randomized Optimal Design of Parallel Manipulators. IEEE, Transactions on Automation Science and Engineering, Vol. X, No. X, $\mathrm{XX}$. 
Arsenault, M. Boudreau, R. (2004). The Synthesis of Three-Degree-of-Freedom Planar Parallel Mechanisms with Revolute Joints (3-RRR) for an Optimal SingularityFree Workspace. Journal of Robotic Systems, pp. 259-274.

Ottaviano, E., Ceccarelli, M., (2002). Optimal Design of CAPAMAN (Cassino Parallel manipulator) with Prescribed Workspace International Journal Robotic, Vol. 20, pp.159-166.

Laribi, M. A., Romdhane, L., Zeghloul S., (2007). Analysis and dimensional synthesis of the DELTA robot for a prescribed workspace. Mechanism and Machine Theory, (42) 859-870, ELSEVIER.

Lou, Y. J., Liu, G. F., Li, Z. X., (2005), A General Approach for Optimal Design of Parallel Manipulators, IEEE, Transactions on Automation Science and Engineering, Vol. X, No. X, XX.

Clavel, R., (1991), Conception d'un Robot Parallele Rapide a 4 Degres de Liberte. Ecole Polytechnique Federale de Lausanne, These pour l'obtention du Grade de Docteur es Sciences Techniques, pág. $10-43$.

Selvakumar, A., et al., (2009). Simulation and Workspace Analisys of a Tripod Parallel Manipulator. World Academy of Science, Engineering and Technology.

Velázquez, M., (2003). Estudio y Análisis del Espacio de Trabajo de un Manipulador Paralelo de 3 Grados de Libertad. Tesis de licenciatura. Universidad Autónoma de Querétaro, Centro Universitario.
Saravia, D., et al., (2009). Revisión del Estado del Arte de Manipuladores Paralelos. Scientia et Technica, Vol. XV, pp. 81-86.

Taha, H., (2004). Investigación de Operaciones, $7^{\mathrm{a}}$. Edición, Pearson educación, México, pp. 1-10.

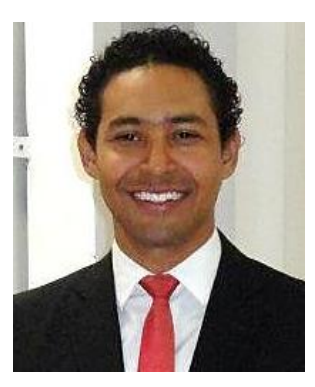

Albert Lester Balmaceda
Santamaría se graduó de
Ingeniero Industrial en
Universidad la
Ingeniería (UNI) en Nicaragua, y
obtuvo el título de Maestro en
Tecnología Avanzada. Así
mismo estudia el doctorado en el CICATA, Unidad Querétaro del Instituto Politécnico Nacional bajo autorización de la UNI, en donde se desempeña como profesor de tiempo completo.

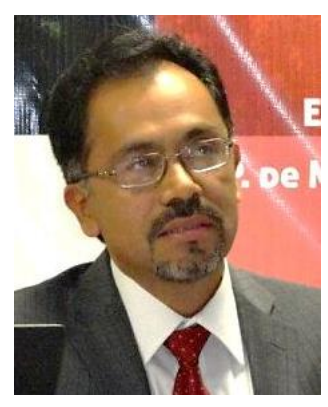

Eduardo Castillo Castañeda se graduó de Ingeniero Mecánico Electricista en la Universidad Autónoma de México y obtuvo el grado de Doctor del Institut National Polytechnique de Grenoble, Francia; en Control Automático. Actualmente, es profesor investigador en el Centro de Investigación en el CICATA, Unidad Querétaro del Instituto Politécnico Nacional. 